\title{
ON AN INTEGRAL OF MARCINKIEWICZ
}

BY

DANIEL WATERMAN

Let $f(\theta)$ be a function of period $2 \pi$ and class $L_{r}, p>1$. By $F(\theta)$ is meant a primitive of $f(\theta)$, i.e.,

$$
F(\theta)=\int_{0}^{\theta} f(x) d x+C .
$$

Marcinkiewicz [3] defined the function

$$
\begin{aligned}
\mu_{r}(\theta) & =\left\{\int_{0}^{2 \pi}\left([F(\theta+t)+F(\theta-t)-2 F(\theta)]^{r / t^{r+1}}\right) d t\right\}^{1 / r} \\
& =\left\{\int_{0}^{\pi} t^{r-1}\left[\frac{F(\theta+t)+F(\theta-t)-2 F(\theta)}{t^{2}}\right]^{r} d t\right\}^{1 / r}
\end{aligned}
$$

and demonstrated the inequalities $\left({ }^{1}\right)$

$$
\begin{array}{rr}
\left\|\mu_{p}\right\|_{p} \leqq A_{p}\|f\|_{p}, & p \geqq 2 \\
\|f\|_{p} \leqq A_{p}\left\|_{1} \mu_{p}\right\|_{p}, & 1<p \leqq 2 .
\end{array}
$$

Since addition of a constant to $f(\theta)$ does not alter $\mu_{p}$, the second inequality clearly requires

$$
\int_{0}^{2 \pi} f(\theta) d \theta=0
$$

Marcinkiewicz raised the question whether $\mu=\mu_{2}$, i.e.,

$$
\mu=\left\{\int_{0}^{2 \pi}[F(\theta+t)+F(\theta-t)-2 F(\theta)]^{2} / t^{3} d t\right\}^{1 / 2},
$$

satisfied the inequality

$$
A_{p}\|f\|_{p} \leqq\|\mu\|_{p} \leqq A_{p}\|f\|_{p}
$$

(the left side requiring, of course, that $\int_{0}^{2 \pi} f d \theta=0$ ). This was affirmatively answered by Zygmund in [7], to which paper the reader is referred for an account of the origin of this problem and its relation to other problems in the theory of Fourier series and in the theory of functions.

Presented to the Society, April 25, 1952; received by the editors February 24, 1956, and, in revised form, May 20, 1957.

(1) Here, as in the sequel, $\|s(t)\|_{p}$ denotes $\left\{\int_{E}|s(t)|^{p} d t\right\}^{1 / p}$, the range $E$ being suitably defined to meet the exigencies of the particular situation. A constant is denoted by an upper-case latin capital, dependence on a parameter being indicated by a subscript; one letter will be used to denote more than one constant. 
The purpose of this paper is to extend this result of Zygmund to the analogously defined function

$$
\mu(\tau)=\left\{\int_{0}^{\infty}[F(\tau+t)+F(\tau-t)-2 F(\tau)]^{2} / t^{3} d t\right\}^{1 / 2},
$$

where $F(\tau)$ is a primitive of a function $f(\tau)$ of class $L_{p}, p>1$ in $(-\infty, \infty)$. Our result is stated explicitly in the following theorem.

Theorem. Let $f(\tau)$ be a function of class $L_{p}, p>1$, in $(-\infty, \infty)$ and $\mu(\tau)$ defined as in (1), then

$$
A_{p}\|f\|_{p} \leqq\|\mu\|_{p} \leqq A_{p}\|f\|_{p} .
$$

It will be necessary to utilize certain concepts and results of the theory of functions, which will be briefly noted here.

If $f(\tau)$ is of class $L_{p}, p>1$, in $(-\infty, \infty)$, its conjugate function $\tilde{f}(\tau)$ is defined by

$$
\frac{1}{\pi} \int_{-\infty}^{\infty} \frac{f(x)}{\tau-x} d x
$$

the integration, naturally, is meant in the principal value sense. The function $\tilde{f}(\tau)$ is of class $L_{p}$, and [4] we have

$$
A_{p}\|\tilde{f}\|_{p} \leqq\|f\|_{p} \leqq A_{p}\|\tilde{f}\|_{p} .
$$

$P(\sigma, \tau)$ will denote the Poisson kernel for the half-plane, $\sigma /\left(\sigma^{2}+\tau^{2}\right)$, and the Poisson integral

$$
\phi(s)=\phi(\sigma+i \tau)=\frac{1}{\pi} \int_{-\infty}^{\infty}(f(x)+i \tilde{f}(x)) P(\sigma, \tau-x) d x
$$

is an analytic function in $R(s)>0$ and is in class $\mathfrak{K}_{p}$, i.e., the integral

$$
\int_{-\infty}^{\infty}|\phi(\sigma+i \tau)|^{p} d \tau
$$

is uniformly bounded in $0<\sigma<\infty$. If

$$
\|\phi(\sigma+i \tau)\|_{p}=\left\{\int_{-\infty}^{\infty}|\phi(\sigma+i \tau)|^{p} d \tau\right\}^{1 / p}
$$

then $\|\phi\|_{p}$ will denote $\|\phi(0+i \tau)\|_{p}$. It may be shown that

$$
\|\phi(\sigma+i \tau)\|_{p} \rightarrow 0
$$

as $\sigma \rightarrow \infty$, continuously and monotonically, that 


$$
\begin{gathered}
|\phi(\sigma+i \tau)|<C \sigma^{-1 / p}\|\phi\|_{p}, \\
\left|\phi^{\prime}(\sigma+i \tau)\right|<C \sigma^{-(1+p) / p}\|\phi\|_{p},
\end{gathered}
$$

and also that for $\delta>0$,

$$
\lim _{\rho \rightarrow \infty}\left|\phi\left(\delta+\rho e^{i \theta}\right)\right|=0, \quad-\pi / 2 \leqq \theta \leqq \pi / 2,
$$

uniformly in $\theta$ [2].

We shall list several lemmas to which we shall refer later.

Let

$$
\Phi(\tau)=\sup _{r \in R^{s}}|\phi(s)|
$$

where

$$
R_{\tau}=\left[s \mid s=i \tau+\rho e^{i \theta}, \rho>0,-\alpha<\theta<\alpha<\pi / 2\right] .
$$

Lemma 1. If $\phi$ is in class $\mathfrak{H}_{p}, p>1$, then

$$
\|\Phi\|_{p} \leqq A_{p}\|\phi\|_{p} .
$$

This result is due to Hardy and Littlewood [1] in the case of the circle; the extension to the half-plane is due to E. Trombley [5].

Lemma 2. If $\phi(s)$ is in class $\mathfrak{H}_{p}, p \geqq 1$, then

$$
\phi^{\prime}(s)=o(1)
$$

as $|s| \rightarrow \infty$ uniformly in every half-plane $\sigma>\alpha>0$.

A proof of this is to be found in a paper of this author [6].

LEMma 3. If

$$
g(\tau)=g(\tau ; \phi)=\left\{\int_{0}^{\infty} \sigma\left|\phi^{\prime}(\sigma+i \tau)\right|^{2} d \sigma\right\}^{1 / 2}
$$

and $\phi(s)$ is in class $\mathfrak{F}_{p}, p \geqq 1$, then

$$
\|g\|_{p} \leqq A_{p}\|\phi\|_{p}
$$

Lemma 4. If

$$
g^{*}(\tau)=g^{*}(\tau ; \phi)=\left\{\frac{1}{\pi} \int_{0}^{\infty} \sigma d \sigma \int_{-\infty}^{\infty}|\phi(\sigma+i \beta)|{ }^{2} P(\sigma, \tau-\beta) d \beta\right\}^{1 / 2},
$$

and $\phi(s)$ is in class $\mathfrak{F}_{p}, p>1$, then

$$
\left\|g^{*}\right\|_{p} \leqq A_{p}\|\phi\|_{p}
$$


These results (together with their converses, which are not required here) are also to be found in [6].

We demonstrate first that

$$
\|f\|_{p} \leqq A_{p}\|\mu\|_{p}
$$

Let

$$
F(\tau)=\int_{c}^{r} f(t) d t
$$

Then

$$
f(\sigma, \tau)=\frac{1}{\pi} \int_{-\infty}^{\infty} f(t) P(\sigma, \tau-t) d t=\frac{1}{\pi} \int_{-\infty}^{\infty} F(t) P_{\tau}(\sigma, \tau-t) d t
$$

and

$$
f_{\tau}(\sigma, \tau)=\frac{1}{\pi} \int_{-\infty}^{\infty} F(t) P_{\tau \tau}(\sigma, \tau-t) d t .
$$

We note that $P_{\tau \tau}(\sigma, \tau)$ is even and that $\int_{0}^{\infty} P_{\tau \tau} d \tau=0$. Thus we may write

$$
\begin{aligned}
f_{\tau}(\sigma, \tau) & =\frac{1}{\pi} \int_{-\infty}^{\infty} F(\tau+t) P_{t t}(\sigma, t) d t \\
& =\frac{1}{2 \pi} \int_{-\infty}^{\infty}[F(\tau+t)+F(\tau-t)-2 F(\tau)] P_{t \iota}(\sigma, t) d t .
\end{aligned}
$$

Let us fix $\tau$. We write

$$
F(\tau+t)+F(\tau-t)-2 F(\tau)=\xi(t) .
$$

Consider

$$
\sigma\left|f_{\tau}(\sigma, \tau)\right|^{2} \leqq A \sigma\left|\int_{0}^{\infty} \xi(t) P_{t t}(\sigma, t) d t\right|^{2}
$$

A simple computation shows that for $|t|<\sigma$

$$
\left|P_{t t}\right|<C \sigma^{-3}
$$

and for $|t|>\sigma$

$$
\left|P_{t t}\right|<C \sigma t^{-4}
$$

Thus

$$
\sigma\left(\int_{0}^{\sigma} \xi P_{t t} d t\right)^{2}<C \sigma^{-5}\left(\int_{0}^{\sigma}|\xi| d t\right)^{2} \leqq C \sigma^{-4} \int_{0}^{\sigma}|\xi|^{2} d t
$$

and 


$$
\sigma\left(\int_{\sigma}^{\infty} \xi P_{t \imath} d t\right)^{2}<C \sigma^{3}\left(\int_{0}^{\infty}|\xi| t^{-4} d t\right)^{2} \leqq C \int_{\sigma}^{\infty}|\xi|^{2} t^{-4} d t
$$

Integrating with respect to $\sigma$,

$$
\int_{0}^{\infty} \sigma^{-4} d \sigma \int_{0}^{\sigma}|\xi|^{2} d t \leqq \int_{0}^{\infty}|\xi|^{2}\left(\int_{t}^{\infty} \sigma^{-4} d \sigma\right) d t=C \mu^{2}(\tau)
$$

and

$$
\int_{0}^{\infty} \sigma d \sigma \int_{0}^{\infty}|\xi|^{2} t^{-4} d t \leqq \int_{0}^{\infty}|\xi|^{2} t^{-4}\left(\int_{0}^{t} d \sigma\right) d t=\mu^{2}(\tau) .
$$

Hence, if $w(\tau)$ denotes

$$
\left\{\int_{0}^{\infty} \sigma\left|f_{\tau}(\sigma, \tau)\right|^{2} d \sigma\right\}^{1 / 2}
$$

we have shown that

$$
w(\tau) \leqq C \mu(\tau) .
$$

We shall now demonstrate that

$$
\|f\|_{p} \leqq A_{p}\|w\|_{p}
$$

and thus the required inequality will be established.

We assume a right translation of the vertical axis so that $f(\tau)$ assumes its original values on $R(s)=-\eta, \eta>0$.

We define

$$
f^{*}(\tau)=f_{N}^{*}(\tau)=|f|^{p-1}(\operatorname{signum} f) \chi_{N}(\tau)
$$

where

$$
\chi_{N}(\tau)=\left\{\begin{aligned}
1, & |\tau|<N-1 \\
0, & |\tau|>N \\
>0, & \leqq 1, \text { and is continuous elsewhere. }
\end{aligned}\right.
$$

The following notation is adopted:

$$
\begin{aligned}
& \phi=u+i v \text { is the analytic function associated with } f, \\
& \psi=u^{*}+i v^{*} \text { is the analytic function associated with } f^{*}, \\
& \gamma=g(\tau ; \psi) .
\end{aligned}
$$

Consider

$$
\left.\left.\int_{\epsilon}^{K} \sigma\left(u u^{*}\right)_{\sigma \sigma} d \sigma=\sigma\left(u u^{*}\right)_{\sigma}\right]_{\epsilon}^{K}-\left(u u^{*}\right)\right]_{\epsilon}^{K} \text {. }
$$


Using a procedure identical with that given in [6], where this device is employed in proving our theorem on $g(\tau)$, we may show that

$$
\int_{0}^{K} \sigma\left(u u^{*}\right)_{\sigma \sigma} d \sigma=u(0, \tau) u^{*}(0, \tau)+o(1) \frac{K}{K^{2}+\tau^{2}}
$$

for large $K$.

Further we may show that for $\mathfrak{n}>N$

$$
\int_{-N}^{N} f f^{*} d \tau=\int_{-\infty}^{\infty} f f^{*} d \tau \leqq 2 \int_{0}^{K} \sigma\left|\int_{-\mathscr{N}}^{\Re}\left(u_{\tau} u_{\tau}^{*}+u_{\sigma} u_{\sigma}^{*}\right) d \tau\right| d \sigma+K \epsilon(\mathfrak{T})+\epsilon(K)
$$

where $\epsilon(y)$ denotes a function $o(1)$ as $y \rightarrow \infty$.

We shall show that

$$
\int_{-\Re}^{\Re}\left(u_{\sigma} u_{\sigma}^{*}-u_{\tau} u_{\tau}^{*}\right) d \tau=o(1)
$$

as $\mathfrak{N} \rightarrow \infty$ for $\sigma>0$, that it is bounded by a function of class $L_{1}$, and hence

$$
\int_{-\infty}^{\infty} f f^{*} d \tau \leqq 4 \int_{0}^{K} \sigma\left|\int_{-\mathscr{T}}^{\Re} u_{\tau} u_{\tau}^{*} d \tau\right| d \sigma+K^{2} \epsilon(\mathfrak{T})+\epsilon(K) .
$$

We note that $\left(u_{\sigma} u_{\sigma}^{*}-u_{\tau} u_{\tau}^{*}\right)=R\left(\phi^{\prime} \phi^{\prime}\right)$. Consider the rectangle $\Delta$ with edges

$$
\begin{aligned}
& \alpha:(\sigma-i \mathfrak{N}, \sigma+i \mathfrak{I}), \quad \beta:(\sigma+i \mathfrak{I}, \sigma+\nu+i \mathfrak{N}), \\
& \gamma:(\sigma+\nu-i \mathfrak{N}, \sigma+\nu+i \mathfrak{N}), \quad \delta:(\sigma-i \mathfrak{T}, \sigma+\nu+i \mathfrak{\Re}) .
\end{aligned}
$$

Clearly

$$
\mathscr{g}\left(\int_{\Delta}\left(\phi^{\prime} \psi^{\prime}\right) d s\right)=0
$$

Thus

$$
\left|\int_{-\mathscr{N}}^{\Re}\left(u_{\sigma} u_{\sigma}^{*}-u_{\tau} u_{\tau}^{*}\right) d \tau\right| \leqq \int_{\gamma}\left|\phi^{\prime} \psi^{\prime}\right| d \tau+\int_{\beta, \delta}\left|-u_{\sigma} u_{\tau}^{*}+v_{\sigma} u_{\sigma}^{*}\right| d \sigma .
$$

We have

$$
\begin{aligned}
\left|u_{\tau}^{*}\right| & \leqq C \int_{-\mathscr{N}}^{\mathscr{N}} \frac{\sigma(\tau-t)}{\left(\sigma^{2}+(\tau-t)^{2}\right)^{2}} d t \\
& \leqq N C \sigma \frac{|\tau|+\mathscr{N}}{\left(\sigma^{2}+(|\tau|-N)^{2}\right)^{2}} \\
& =o(1) \text { as }|\tau| \rightarrow \infty, \text { uniformly in } \sigma,
\end{aligned}
$$

and similarly 


$$
\begin{aligned}
\left|u_{\sigma}^{*}\right| & \leqq C \int_{-N}^{N} \frac{\left|(\tau-t)^{2}-\sigma^{2}\right|}{\left(\sigma^{2}+(\tau-t)^{2}\right)^{2}} d t \\
& \leqq C \int_{-N}^{N} \frac{1}{\sigma^{2}+(\tau-t)^{2}} d t \\
& \leqq C N \frac{1}{\sigma^{2}+(|\tau|-N)^{2}} \\
& =o(1) \text { as }|\tau| \rightarrow \infty, \text { uniformly in } \sigma .
\end{aligned}
$$

Thus

$$
\int_{\beta}\left|-u_{\sigma} u_{\tau}^{*}+v_{\sigma} u_{\sigma}^{*}\right| d \sigma \leqq \epsilon(\mathfrak{T}) \int_{\beta}\left|\phi^{\prime}\right| d \sigma=\epsilon(\mathfrak{T})
$$

since $\phi^{\prime}=O\left(\sigma^{-(1+p) / p}\right)$ as $\sigma \rightarrow \infty$ and is bounded in the half-plane (Lemma 2), and similarly for the integral over segment $\delta$. Also

$$
\int_{\gamma}\left|\phi^{\prime} \psi^{\prime}\right| d \tau \leqq C \int_{-\Re}^{\Re}(\Phi / \nu) \cdot(\Psi / \nu) d \tau \leqq \frac{C}{\nu^{2}}\|\Phi\|_{p}\|\Psi\|_{p^{\prime}}=\frac{C}{\nu^{2}} .
$$

If $\nu=1 / \mathscr{N}$ we have at once that

$$
\int_{-\mathscr{N}}^{\mathfrak{N}}\left(u_{\sigma} u_{\sigma}^{*}-u_{\tau} u_{\tau}^{*}\right) d t=o(1)
$$

as $\Re \rightarrow \infty$ independent of $\sigma$.

Since

$$
\begin{aligned}
\left|\int_{-\Re}^{\Re}\left(u_{\sigma} u_{\sigma}^{*}-u_{\tau} u_{\tau}^{*}\right) d \tau\right| & \leqq \int_{-\Re}^{\Re}\left|\phi^{\prime} \psi^{\prime}\right| d \tau \\
& \leqq\|\Phi\|\left\|_{p}\right\| \Psi \|_{p^{\prime}} \cdot \frac{1}{\sigma^{2}} \\
& =C \cdot \frac{1}{\sigma^{2}}
\end{aligned}
$$

for large $\sigma$, and by the previous argument is bounded for $\sigma$ small and $\Re$ sufficiently large, clearly it is dominated by a function of class $L_{1}$, and our estimate of $\int_{-\infty}^{\infty} f h d \tau$ has been validated.

Allowing $\mathfrak{X}$ and then $K$ to approach infinity, we have

$$
\int_{-\infty}^{\infty} f h d \tau \leqq C \int_{0}^{\infty} \sigma \int_{-\infty}^{\infty}\left|u_{\tau} u_{\tau}^{*}\right| d_{\tau} d \sigma
$$

and so 


$$
\begin{aligned}
\int_{-\infty}^{\infty} f h d \tau & \leqq C \int_{0}^{\infty} \sigma \int_{-\infty}^{\infty}\left|u_{\tau} \psi^{\prime}\right| d \tau d \sigma \\
& \leqq C \int_{-\infty}^{\infty}\left(\int_{0}^{\infty} \sigma\left|u_{\tau}\right|^{2} d \sigma\right)^{1 / 2}\left(\int_{0}^{\infty} \sigma\left|\psi^{\prime}\right|^{2} d \sigma\right)^{1 / 2} d \tau \\
& =C \int_{-\infty}^{\infty} w(\tau) \gamma(\tau) d \tau \\
& \leqq C\|w\|_{p} \cdot\|\gamma\|_{p^{\prime}} \\
& \leqq A_{p}\|w\|_{p}\|h\|_{p^{\prime}}
\end{aligned}
$$

by Lemma 3. Allowing $N$ to approach infinity we have

$$
\int_{-\infty}^{\infty}|f|^{p} d \tau \leqq A_{p}\|w\|_{p}\|f\|_{p}^{p-1}
$$

or

$$
\|f\|_{p} \leqq A_{p}\|w\|_{p^{\prime}}
$$

We recall, however, that a right translation of the vertical axis has been made. For $\eta>0$,

$$
w(\tau ; f(\sigma+\eta+i \tau)) \leqq w(\tau ; f(\sigma+i \tau))
$$

and

$$
\|f(\eta+i \tau)\|_{p} \rightarrow\|f(\tau)\|_{p}
$$

as $\eta \rightarrow 0$. Therefore

$$
\|f\|_{p} \leqq A_{p}\|w\|_{p}
$$

where $f$ is as originally defined and $w$ corresponds to it. Since

$$
w \leqq C_{\mu}
$$

our inequality has been established.

We turn now to the inequality

$$
\|\mu\|_{p} \leqq A_{p}\|f\|_{p} .
$$

First we define (analogously to Zygmund [7])

$$
\begin{aligned}
F(\sigma, t) & =\int_{0}^{t} f(\sigma+i \tau) d \tau \\
F_{1}(\sigma, t) & =F(\sigma, \tau+t)+F(\sigma, \tau-t)-2 F(\sigma, \tau), \\
F_{1}(t) & =F_{1}(0, t), \\
f_{1}(\sigma, t) & =\partial F_{1}(\sigma, t) / \partial t=f(\sigma, \tau+t)-f(\sigma, \tau-t) .
\end{aligned}
$$


We are then able to write

$$
\mu(\tau)=\left\{\int_{0}^{\infty}\left(\left[F_{1}(t)\right]^{2} / t^{3}\right) d t\right\}^{1 / 2} .
$$

Let

$$
F_{1}(t)=\left\{F_{1}(0, t)-F_{1}\left(\sigma_{t}, t\right)\right\}+\left\{F_{1}\left(\sigma_{t}, t\right)-F_{1}\left(\sigma_{t}, 0\right)\right\}=V+W .
$$

Consider the function $W$. Proceeding in a manner similar to that of Zygmund [7], we have

$$
W^{2} \leqq\left\{\int_{0}^{t}\left|f_{1}\left(\sigma_{t}, u\right)\right| d u\right\}^{2} \leqq t^{3} \int_{-t}^{t} \mid f_{v}\left(\sigma_{t}, \tau+\left.v\right|^{2} d v\right.
$$

Letting $\phi(\sigma+i \tau)$ again be the analytic function associated with $f(\tau)$, and $\sigma_{t}=t$,

$$
\begin{aligned}
\int_{0}^{\infty} t^{-3} W^{2}(t) d t & \leqq \int_{0}^{\infty} d \sigma \int_{-\sigma}^{\sigma}\left|\phi^{\prime}(\sigma+i(\tau+v))\right|^{2} d v \\
& \leqq A g^{*^{2}}(\tau) .
\end{aligned}
$$

By an integration by parts

$$
|V|=\left|F_{1}(0, t)-F_{1}\left(\sigma_{t}, t\right)\right| \leqq \int_{0}^{\sigma_{t}} \sigma\left|F_{1_{\sigma \sigma}}\right| d \sigma+\sigma_{t}\left|F_{1_{\sigma}}\left(\sigma_{t}, t\right)\right|=V_{1}+V_{2} \text {. }
$$

We write

$$
\begin{aligned}
V_{1} & \leqq \int_{0}^{\sigma_{t}} \sigma\left|f_{\tau}(\sigma, \tau+t)\right| d \sigma+\int_{0}^{\sigma_{t}} \sigma\left|f_{\tau}(\sigma, \tau-t)\right| d \sigma+\int_{0}^{\sigma_{t}} \sigma\left|f_{\tau}(\sigma, \tau)\right| d \sigma \\
& =V_{11}+V_{12}+V_{13} .
\end{aligned}
$$

Letting $\sigma_{t}=t$, we have

$$
\begin{aligned}
\int_{0}^{\infty} V_{11}^{2} t^{-3} d t & \leqq \int_{0}^{\infty} t^{-2} d t \int_{0}^{t} \sigma\left|f_{\tau}(\sigma, \tau+t)\right|^{2} d \sigma \\
& \leqq \int_{0}^{\infty} t^{-2} d t \int_{0}^{t} \sigma\left|\phi^{\prime}(\sigma+i(\tau+t))\right|^{2} d \sigma \leqq A g^{*^{2}}(\tau)
\end{aligned}
$$

and similarly

$$
\int_{0}^{\infty} V_{12}^{2} t^{-3} d t \leqq A g^{*^{2}}(\tau), \quad \int_{0}^{\infty} V_{13}^{2} t^{-3} d t \leqq g^{2}(\tau) .
$$

Thus 


$$
\int_{0}^{\infty} V_{1}^{2} t^{-3} d t \leqq A\left\{g^{2}(\tau)+g^{*^{2}}(\tau)\right\}
$$

Examining $V_{2}$ now,

$$
\begin{aligned}
V_{2} & =\sigma_{t}\left|F_{1_{\sigma}}\left(\sigma_{t}, t\right)\right| \\
& =\sigma_{t}\left|F_{\sigma}\left(\sigma_{t}, \tau+t\right)+F_{\sigma}\left(\sigma_{t}, \tau-t\right)-2 F_{\sigma}\left(\sigma_{t}, \tau\right)\right| .
\end{aligned}
$$

Note that

$$
F_{\sigma}(\sigma, u)=\int_{0}^{u} f_{\sigma}(\sigma, x) d x=\int_{0}^{u} \tilde{f}_{x}(\sigma, x) d x=\tilde{f}(\sigma, u)-\tilde{f}(\sigma, 0) .
$$

Hence

$$
V_{2}=\sigma_{t}\left|\tilde{f}\left(\sigma_{t}, \tau+t\right)+\tilde{f}\left(\sigma_{t}, \tau-t\right)-2 \tilde{f}\left(\sigma_{t}, \tau\right)\right| \leqq \sigma_{t} \int_{-t}^{t} \mid \tilde{f}_{\tau}\left(\sigma_{t}, \tau+v\right) d v
$$

and

$$
V_{2}^{2} \leqq A \sigma_{t}^{2} \cdot t \int_{-t}^{t}\left|\phi^{\prime}\left(\sigma_{t}+i(\tau+v)\right)\right|^{2} d v
$$

Letting $\sigma_{t}=t$,

$$
\int_{0}^{\infty} V_{2}^{2} t^{-3} d t \leqq A \int_{0}^{\infty} d \sigma \int_{-\sigma}^{\sigma}\left|\phi^{\prime}(\sigma+i(\tau+v))\right|^{2} d v \leqq A g^{*^{2}}(\tau) .
$$

We have then

$$
\mu^{2}(\tau) \leqq A\left\{g^{2}(\tau)+g^{*^{2}}(\tau)\right\},
$$

and by Lemmas 3 and 4

$$
\|\mu\|_{p} \leqq A_{p}\|f\|_{p}
$$

for $p>1$, which completes the proof of our theorem.

\section{REFERENCES}

1. G. H. Hardy and J. E. Littlewood, A maximal theorem with function-theoretic applications, Acta Math. vol. 54 (1930) pp. 81-116.

2. E. Hille and J. D. Tamarkin, On the absolute integrability of Fourier transforms, Fund. Math. vol. 25 (1935) pp. 329-352.

3. J. Marcinkiewicz, Sur quelques intégrales du type de Dini, Ann. Soc. Polon. Math. vol. 17 (1938) pp. 42-50.

4. M. Riesz, Sur les séries conjugées, Math. Z. vol. 27 (1927) pp. 218-244.

5. E. Trombley, Unpublished manuscript.

6. D. Waterman, On functions analytic in a half-plane, Trans. Amer. Math. Soc. vol. 81 (1956) pp. 167-194.

7. A. Zygmund, On certain integrals, Trans. Amer. Math. Soc. vol. 55 (1944) pp. 170-204.

Purdue University,

LAFAYETTE, IND. 